\title{
BMJ Open Access all areas? An area-level analysis of accessibility to general practice and community pharmacy services in England by urbanity and social deprivation
}

To cite: Todd A, Copeland A, Husband $A$, et al. Access all areas? An area-level analysis of accessibility to general practice and community pharmacy services in England by urbanity and social deprivation. BMJ Open 2015:5:e007328.

doi:10.1136/bmjopen-2014007328

- Prepublication history for this paper is available online. To view these files please visit the journal online (http://dx.doi.org/10.1136/ bmjopen-2014-007328).

Received 28 November 2014 Revised 23 February 2015 Accepted 4 March 2015

CrossMark

For numbered affiliations see end of article.

Correspondence to

Dr Adam Todd;

adam.todd@durham.ac.uk

\section{ABSTRACT}

Objectives: (1) To determine the percentage of the population in England that has access to a general practitioner (GP) premises within a 20 min walk (the accessibility); (2) explore the relationship between the walking distance to a GP premises and urbanity and social deprivation and (3) compare accessibility of a GP premises to that of a community pharmacy-and how this may vary by urbanity and social deprivation. Design: This area-level analysis spatial study used postcodes for all GP premises and community pharmacies in England. Each postcode was assigned to a population lookup table and Lower Super Output Area (LSOA). The LSOA was then matched to urbanity (urban, town and fringe, or village, hamlet and isolated dwellings) and deprivation decile (using the Index of Multiple Deprivation score 2010).

Primary outcome measure: Living within a $20 \mathrm{~min}$ walk of a GP premises.

Results: Overall, $84.8 \%$ of the population is estimated to live within a 20 min walk of a GP premises: $81.2 \%$ in the most affluent areas, $98.2 \%$ in the most deprived areas, $94.2 \%$ in urban and $19.4 \%$ in rural areas. This is consistently lower when compared with the population living within a 20 min walk of a community pharmacy.

Conclusions: Our study shows that the vast majority of the population live within a 20 min walk of a GP premises, with higher proportions in the most deprived areas-a positive primary care law. However, more people live within a 20 min walk of a community pharmacy compared with a GP premises, and this potentially has implications for the commissioning of future services from these healthcare providers in England.

\section{BACKGROUND}

Primary care is a vital component of the healthcare system in the developed world. In the UK, over $90 \%$ of all contacts with
Strengths and limitations of this study

- Our study is the first to systematically explore the spatial distribution of general practitioner (GP) premises in England using walking distances, and the first to make a direct comparison with the accessibility of community pharmacies.

- A key strength of this study is that we examined accessibility by walking distance; the cost of driving and using public transport can be significant barriers to travel.

- A possible limitation is that not all patients will be registered with their nearest GP premises; we did not seek to calculate average walking distances to patients registered to a particular GP premises, or consider 'catchment' areas of GP premises.

healthcare professionals occur in general practitioner (GP) premises, resulting in over 300 million consultations per year. ${ }^{1}$ These consultations are typically GP or nurse-led, and are used to treat acute illness, manage long-term conditions, and promote good health. As such, many GP premises offer public health services, including vaccination clinics, health check-ups and smoking cessation programmes. Furthermore, since the establishment of Clinical Commissioning Groups, GPs have been given an explicit responsibility to tackle health inequalities and under the Health and Social Care Act of 2012, while NHS England is tasked with reducing inequalities around healthcare outcomes. GP premises-and the services they offer-therefore, play a crucial role in maintaining and promoting population health and reducing health inequalities.

As access to healthcare is a well-established social determinant of health, and considering 
the concept of the inverse care law ${ }^{2}$ - where "the availability of good medical care tends to vary inversely with the need for it in the population served"-a number of studies have previously explored the relationship between GP access and social deprivation. These studies have shown that access to GP services in more deprived areas tends to be as good, if not better than more affluent areas. ${ }^{3}{ }^{4}$ However, despite these findings, and although life expectancy has risen overall, the gap in life expectancy between the most deprived and most affluent communities has widened-representing a significant challenge to our society. ${ }^{5}$ While healthcare access is only one element in the complex aetiology of health inequalities, ${ }^{5}$ significantly, more people die from conditions associated with modifiable risk factors such as smoking, alcohol and obesity in deprived communities, than in more affluent ones. ${ }^{6-8}$ A report by the King's Fund ${ }^{9}$ suggests that a number of factors within general practice may be contributing to this: first, recruiting GPs to work in areas of high deprivation is challenging; and second, while policymakers advocate the use of community engagement to tackle the wider determinants of health, it is not clear if GPs feel equipped or motivated to do this; in addition, some GPs feel that tackling issues around public health and health inequalities, such as smoking or obesity, could damage the doctor-patient relationship if done at the wrong time in the consultation process.

In response to these challenges, there is a growing emphasis on using other primary care providers to deliver healthcare services to the population-with one example being the community pharmacy. Indeed, community pharmacies, as part of the NHS Contractual Framework, are commissioned by local authorities to provide services that are designed to help address public health priority conditions, including smoking, alcohol misuse and obesity. ${ }^{10}$ Significantly, recent work has also shown that, in England, approximately $90 \%$ of the population can access a community pharmacy within a $20 \mathrm{~min}$ walk and, in the areas of highest deprivation, the value increases to almost $100 \%$ - the so-called positive pharmacy care law. ${ }^{11}$ Working alongside GP premises, community pharmacies may therefore be in a unique position to deliver healthcare interventions, particularly in areas of greatest deprivation; they also offer the advantage that, at present, appointments are not needed to obtain healthcare advice. However, despite this potential, and given the wider issues of healthcare access as a social determinant of health, it is not known how the accessibility of GP premises compares with the accessibility of community pharmacies in England. This study is, therefore, the first to (1) determine the percentage of the population in England that has access to a GP premises within a $20 \mathrm{~min}$ walk (the accessibility); (2) explore any relationship between the walking distance to a GP premises and urbanity and social deprivation and(3) compare a GP premises accessibility to that of a community pharmacyand how this may vary by urbanity and social deprivation.
METHODS

Definitions

GP premises: a location where a GP actively consults with patients; we included 'satellite' surgeries in the data analysis, while primary care NHS Walk-in-Centres were excluded.

GP: a licensed medical graduate who gives personal, primary and continuing care to individuals, families and a practice population irrespective of age, sex and illness. ${ }^{12}$

Community pharmacy: registered with the General Pharmaceutical Council ${ }^{13}$ as premises for the compounding, procurement, storage and distribution of medicines and appliances; we excluded premises that were solely registered as internet pharmacies in the analysis.

LSOA: lower super output area: geographic areas in England comprising approximately 1500 residents and 650 households. They are designed to be of a reasonably compact shape while being representative of social homogeneity.

Urban/rural classification: categorises each LSOA into three settlement types: urban (where the population is over 10000 people), town and fringe (part of a settlement with less than 10000 people) or village, hamlet and isolated dwellings. ${ }^{14}$ Urbanity was included because rural areas are often disadvantaged in accessing healthcare services-the so called 'distance decay' effect whereby GP consultation rates decline as the distance from the GP surgery increases, and in rural areas this can be exacerbated by a lack of suitable transport. ${ }^{15-17}$

Deprivation decile: the term 'deprivation decile' was used to group the population into 10 equally spaced ordinal categories according to a particular level of deprivation. $^{18}$

\section{Study design}

This study used geographical information systems and generalised linear models to explore the relationship between spatial access to a GP premises in England, deprivation and urbanity, and compares these data to that of a community pharmacy.

\section{Data and variables}

Data were obtained from the Fuse Geo-Health Care Access Database. ${ }^{19}$ This database contains data on the address and postcode of each GP premises in England, the postcode of each community pharmacy (matched to their corresponding coordinates using the Office of National Statistics postcode directory, 2012) $;^{20}$ the coordinates for each postcode in England (also from the Office of National Statistics postcode directory, 2012-matched to a population lookup table and the LSOA for each postcode); whether the LSOA is urban, town and fringe or village, hamlet and isolated dwellings from the urban/ rural classification (2005), and the Index of Multiple Deprivation (IMD) score (2010) for each LSOA (from the Office of National Statistics). 


\section{Outcome}

The percentage of the population that lived within a 20 min walk of a GP premises by LSOA: measured as a straight-line distance from each GP premises $(1.6 \mathrm{~km}$ buffers) and aggregating the population by postcode within each buffer compared with the LSOA population.

\section{Analysis}

The GP premises for England were mapped using ArcMap, using an average walking speed of 3 miles $(4 \mathrm{~km})$ per hour; ${ }^{21} 1.6 \mathrm{~km}$ buffers were placed around the site to represent a 20 min walk from a GP premises. The population postcodes were clipped to the GP premises buffers. Data on the sum of the population for each LSOA overall and within each GP premises buffer was extracted by LSOA. The percentage of the population with access to a GP premises within a 20 min walk was then calculated by LSOA. Whether or not the LSOA was urban, town and fringe or rural, and the corresponding deprivation score was attached to the data file. Deprivation deciles, based on the IMD for England, were also calculated: the most deprived decile (10) equates to the most deprived $10 \%$ within a population, while the least deprived decile (1) represents the $10 \%$ of a population living in the least deprived circumstances. Each data set was then mapped using Arc GIS. Tables were produced to show the percentage of the population who had access to a GP premises within a 20 min walk by deprivation decile and by urban/rural classification within the deprivation deciles separately. General linear models were used to adjust for rurality within each deprivation decile to produce urban/rural adjusted prevalence rates of the percentage of households with access to a GP premises within a $20 \mathrm{~min}$ walk by LSOA, and the results tabulated.

A causal framework was then used to investigate the impact of area-level deprivation on the association between access to a GP premises and a community pharmacy (based on previous work ${ }^{11}$ ) by fitting three different models to a varying level of complexity. Model 1 is a regression model with access to a GP premises as the outcome variable, and access to a community pharmacy as an explanatory variable. Model 2 contains access to a community pharmacy and area-level deprivation as an explanatory variable, while model 3 contains access to a community pharmacy and area-level deprivation, as well as their interaction as explanatory variables. The percentage change in gradient/slope between access to a GP premises and a community pharmacy from models 1 to 2 was calculated as $100 \times($ model $1-$ model 2$) /$ model 1 .

\section{RESULTS}

\section{GP access}

Data for all the LSOAs in England shows that, overall, $84.8 \%$ of the population lives within a 20 min walk of a GP premises. When considering urbanity, $94.2 \%$ of households in the urban areas live within a 20 min walk of a GP premises, in town and fringe, the figure reduces to $68.1 \%$, while the percentage of those in rural areas with access is reduced to $19.4 \%$.
The distributions of percentages of households with access to a GP premises within a 20 min walk are presented in table 1 (and shown spatially in figures 1 and 2) according to their deprivation decile and urban, town and fringe, and rural areas. There is a significant non-linear association between the deprivation deciles: the least deprived and most deprived households have more access to a GP premises than the households in the middle of the deprivation range.

Overall, the inequalities in access to a GP premises in England range from $1.4 \%$ to $24.4 \%$ less access among the more affluent areas, when compared with the most deprived decile: a positive care law. There are no significant spatial inequalities in access in rural areas or town and fringe area, while urban areas have between $0.8 \%$ and $10.9 \%$ less access when compared with the most deprived decile. Spatial inequalities, therefore, in terms of GP premises accessibility, are much more significant in urban areas.

\section{GP premises and community pharmacy access}

GP premises accessibility was compared with community pharmacy accessibility using previously reported data, ${ }^{11}$ and shows for GP premises, access is lower than community pharmacies across all deprivation deciles, but particularly in the least deprived areas (figure 3). Overall, for GP premises, $84.8 \%$ of the population lives within a 20 min walk, which is $4.4 \%$ lower when compared with the percentage of the population living within a $20 \mathrm{~min}$ walk of a community pharmacy.

General linear models were conducted to examine the relationship between walking distance to a GP premises and community pharmacies, adjusting for deprivation and urbanity for all areas, then for urban, town and fringe, and rural areas (table 2).

There is a significant positive association between community pharmacy accessibility and GP premises accessibility. LSOAs with high percentages of households with access to community pharmacy within a $20 \mathrm{~min}$ walk also have a high percentage of households with access to GP services within a $20 \mathrm{~min}$ walk. However, a $1 \%$ increase in overall community pharmacy access only corresponds to $0.86 \%$ increase in GP premises access, which is an indication of greater community pharmacy accessibility. The gradient between GP premises and community pharmacy access is dependent on the urbanity level-with a higher gradient in urban areas than in village, hamlet and isolated dwellings. The association between GP premises and community pharmacy access is also dependent on deprivation deciles with approximately 5-13\% changes in gradients between GP premises and community pharmacy access after adjusting for deprivation based on all the data, and in urban areas.

\section{DISCUSSION}

\section{Principal findings of this study}

The analysis has shown that $84.8 \%$ of the population in England lives within a 20 min walking distance of a GP 

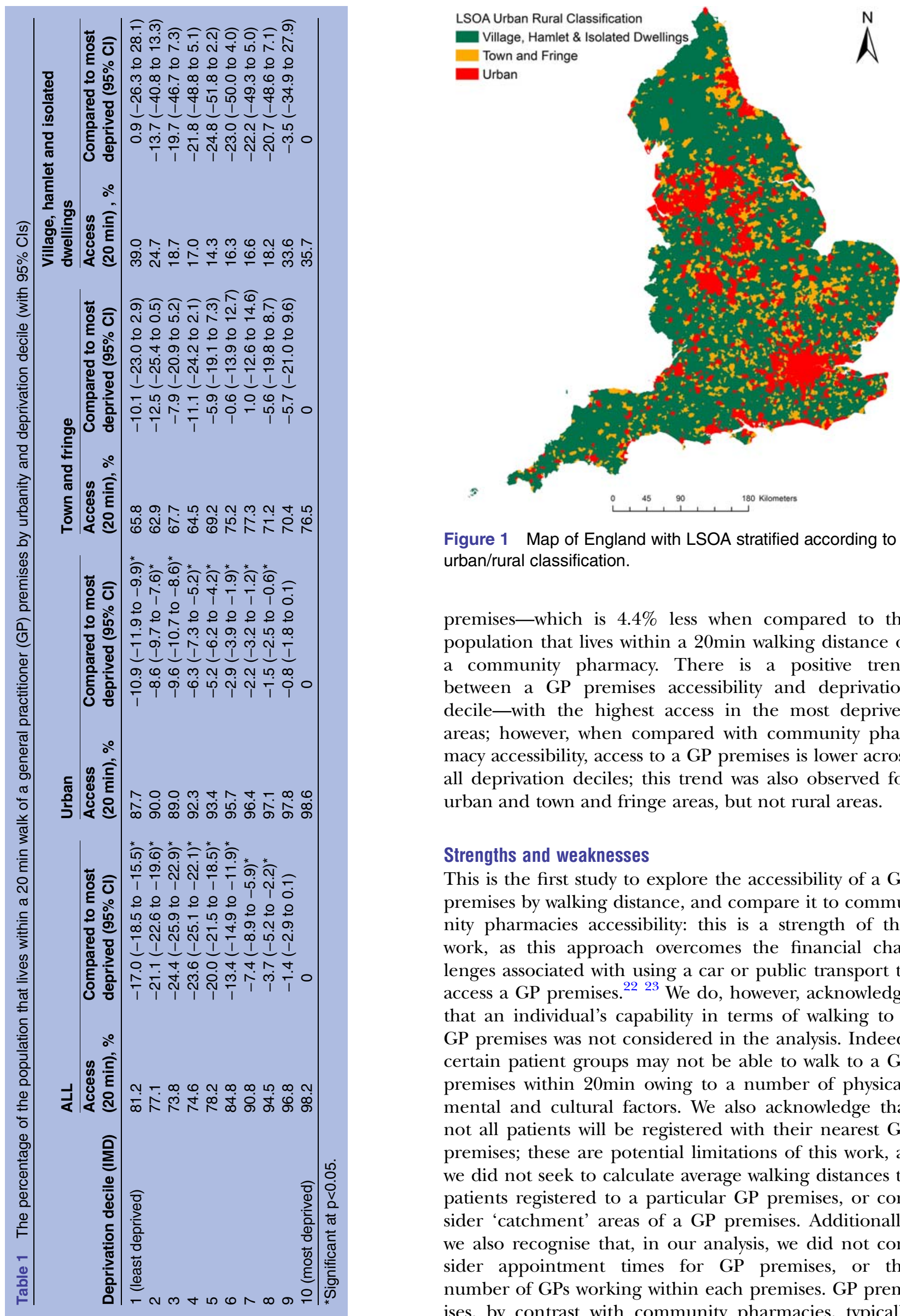

Figure 1 Map of England with LSOA stratified according to urban/rural classification.

premises-which is $4.4 \%$ less when compared to the population that lives within a $20 \mathrm{~min}$ walking distance of a community pharmacy. There is a positive trend between a GP premises accessibility and deprivation decile-with the highest access in the most deprived areas; however, when compared with community pharmacy accessibility, access to a GP premises is lower across all deprivation deciles; this trend was also observed for urban and town and fringe areas, but not rural areas.

\section{Strengths and weaknesses}

This is the first study to explore the accessibility of a GP premises by walking distance, and compare it to community pharmacies accessibility: this is a strength of this work, as this approach overcomes the financial challenges associated with using a car or public transport to access a GP premises. ${ }^{22}{ }^{23}$ We do, however, acknowledge that an individual's capability in terms of walking to a GP premises was not considered in the analysis. Indeed, certain patient groups may not be able to walk to a GP premises within 20min owing to a number of physical, mental and cultural factors. We also acknowledge that not all patients will be registered with their nearest GP premises; these are potential limitations of this work, as we did not seek to calculate average walking distances to patients registered to a particular GP premises, or consider 'catchment' areas of a GP premises. Additionally, we also recognise that, in our analysis, we did not consider appointment times for GP premises, or the number of GPs working within each premises. GP premises, by contrast with community pharmacies, typically 


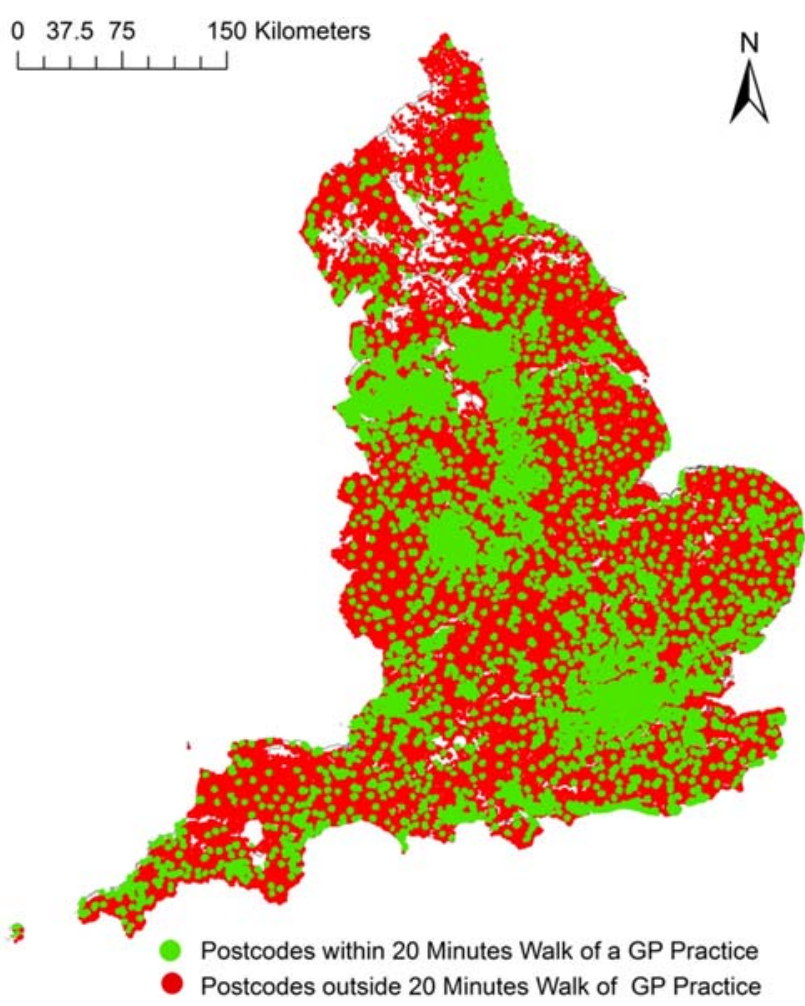

Figure 2 Map of England showing the population within 20 minutes walk of a GP premises.

require a prior appointment before a consultation with a healthcare practitioner. At present, the current waiting time for a non-urgent GP appointment is approximately 9 days, and this figure is expected to increase further in the coming years. ${ }^{24}$ The fact that we did not consider appointment times or the quality of these services are further limitations of our work. From a methodological perspective, we accept that a $20 \mathrm{~min}$ walk has been represented using a straight-line distance from the central point of each postcode to create a buffer. This assumes that people walk in straight lines when, in reality, they are constrained to pathways that curve or are restricted by barriers. Another limitation is the postcodes we used in the analysis: individual postcodes represent approximately 15 addresses, with the coordinates of each postcode being represented using the geographic centroid of each postcode. This could therefore mean (particularly in rural areas) that some households may fall outside of a GP premises buffer.

\section{What is already known}

There have been several studies that have explored the accessibility of GP services, although none have done so by average walking distance, as we have reported herein. For example, Adams and White used the access domain of the IMD 2000 to measure proximity to general practices and markers of socioeconomic deprivation across wards in the north of England. ${ }^{3}$ The work showed that geographical proximity to GP practices was greater in the more deprived wards, compared with more affluent ones, although it was acknowledged that proximity to a GP practice is only one aspect of the provision of good quality care. Similarly, Pearce $e t a t^{t}$ showed that access to community resources-including GP services-was better in deprived areas, compared with more affluent ones. Our work supports these findings and shows that, in England, the majority of the population live within a 20 min walking distance of a GP premises, and this is greatest in areas of high deprivation. GP accessibility by driving distance has also been reported in the literature: Lovett $e t a l^{25}$ investigated GP access according to public and private transport in East Anglia. The work showed that, to access a GP, $10 \%$ of residents needed to travel more than $10 \mathrm{~min}$ by car, while $13 \%$ of the population was unable to attend a GP appointment if they were reliant on solely using public transport.

\section{Implications for policymakers}

This is the first study to systematically explore the spatial distribution of GP premises in England using walking distances, and the first to make a direct comparison with the accessibility of community pharmacies. Our results, therefore, have potential implications for policymakers and commissioners of healthcare services in England, although we accept that just because an individual lives within a 20 min walk of a GP premises or community pharmacy, does not necessarily equate to access. Given the complex aetiology of health inequalities, and that healthcare access is only one social determinant of health, it is evident that policymakers should not solely focus on improving healthcare access as a strategy to
Figure 3 Percentage of the population by deprivation decile with access to a GP premises within a 20-minute walk compared to a community pharmacy.

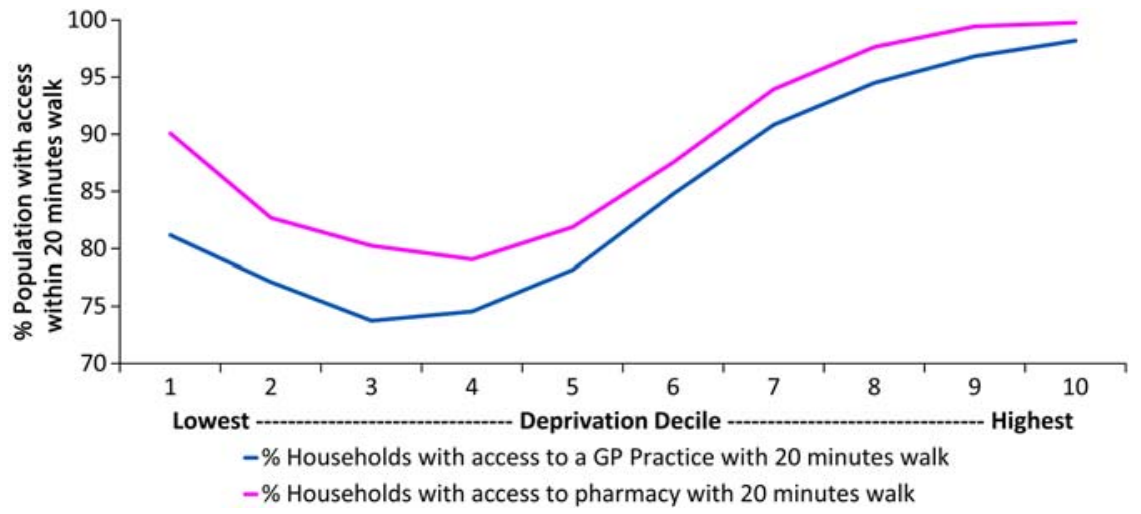




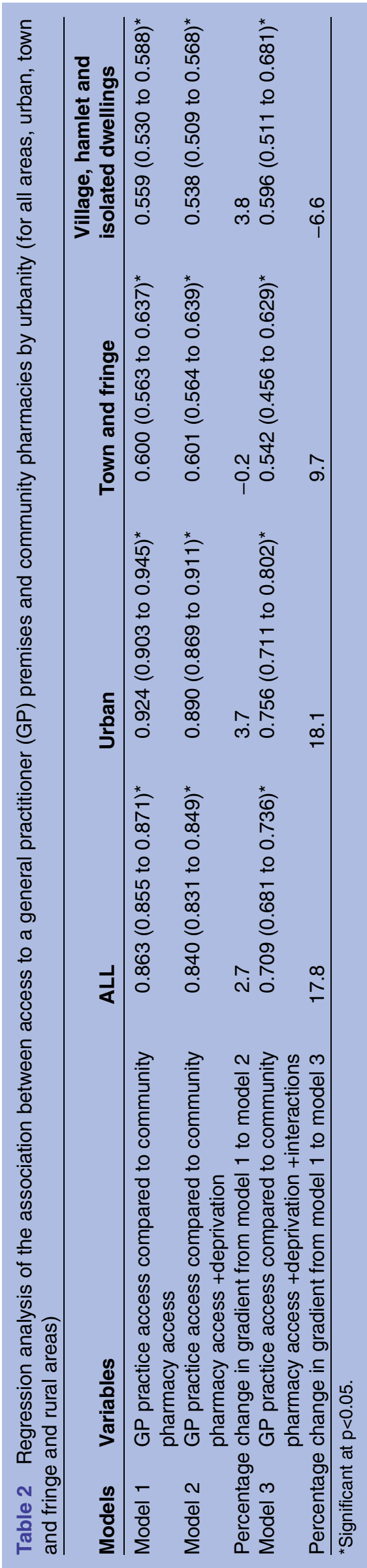

reduce overall inequalities in health. We would, however, urge all policymakers to maintain the level of access to healthcare services in England; a recent study by Barr and colleagues has also shown that increasing the proportion of resources invested in deprived communities was associated with a reduction in absolute health inequalities from causes amenable to healthcare. ${ }^{26}$

In terms of developing services, GP premises and community pharmacies are commissioned-either nationally or locally-to provide public health services. One such example is the administration of influenza vaccinations whereby GPs and community pharmacists offer vaccinations to 'high-risk' groups. Commissioning of these services-particularly those in community pharmacies-has been challenging in some areas. ${ }^{27}$ Our study shows that due to the differences in accessibility-particularly considering deprivation and urbanity-community pharmacies and GP premises could both be used to deliver public health services. It is not yet clear how patients perceive the differences in these providers, but results from qualitative studies suggest, for some services due to the ease of access, community pharmacy is the preferred location. ${ }^{28}$ It is also not understood if there are any differences in patient demographics of those who are accessing services in GP premises or community pharmacies. Further work is, therefore, required to establish who is using these services in these settings to help ascertain if commissioning public health services from these providers can help reduce inequalities in health.

\section{CONCLUSIONS}

In England, the majority of the population live within a 20 min walk of a GP premises-with higher proportions in the most deprived areas (a positive primary care law). However, when compared with community pharmacy accessibility, the percentage of the population that lives within a 20 min walk of a GP premises was significantly lower. This finding has potential implications for the commissioning of future healthcare services from GP premises and community pharmacies in England.

Author affiliations

${ }^{1}$ Division of Pharmacy, School of Medicine, Pharmacy and Health, Durham University, Stockton-on-Tees, UK

${ }^{2}$ Wolfson Research Institute for Health and Wellbeing, Durham University, Stockton-on-Tees, UK

${ }^{3}$ Centre of Health and Inequalities Research (CIHR), Durham University, Durham, UK

${ }^{4}$ Department of Geography, Durham University, Durham, UK

Twitter Follow Adam Todd at @adam.todd138

Contributors AT and CB conceptualised and designed the study and were involved in methodological development, analysis and interpretation. AC, AK and $\mathrm{AH}$ were involved in the development of the methods, data analysis and interpretation. AT led the drafting of the manuscript with input from all authors. All authors approved the final version, and AT has responsibility for its final content.

Funding This research received no specific grant from any funding agency in the public, commercial or not-for-profit sectors. 
Competing interests None declared.

Provenance and peer review Not commissioned; externally peer reviewed.

Data sharing statement All the primary data sources used to compile the database for this study are referenced within the manuscript. We are unable to share the study database, but information related to it is available at (https:// www.dur.ac.uk/wolfson.institute/geohealth/). The final raw analysed data is available by e-mailing AT (adam.todd@durham.ac.uk).

Open Access This is an Open Access article distributed in accordance with the Creative Commons Attribution Non Commercial (CC BY-NC 4.0) license, which permits others to distribute, remix, adapt, build upon this work noncommercially, and license their derivative works on different terms, provided the original work is properly cited and the use is non-commercial. See: http:// creativecommons.org/licenses/by-nc/4.0/

\section{REFERENCES}

1. The Information Centre. General personal medical services: England 1998-2008. Leeds: The Information Centre, 2009.

2. Tudor Hart J. The inverse care law. Lancet 1971;297:405-12.

3. Adams J, White M. Socio-economic deprivation is associated with increased proximity to general practices in England: an ecological analysis. J Public Health 2005;27:80-1.

4. Pearce J, Witten K, Hiscock R, et al. Are socially disadvantaged neighbourhoods deprived of health-related community resources? Int J Epidemiol 2007;36:348-55.

5. Marmot Review. Fair society, healthy lives: the marmot review. London: The Marmot Review, 2010. http://www.instituteofhealth equity.org/projects/fair-society-healthy-lives-themarmot-review (accessed 13 Feb 2015).

6. Alcohol-related deaths in the United Kingdom. Office for National Statistics, 2010. http://www.ons.gov.uk/ons/dcp171778_254061.pdf (accessed 13 Feb 2015).

7. Statistics on smoking: England. The NHS Information Centre for Health and Social Care, 2011. http://www.ic.nhs.uk/pubs/smoking11 (accessed 13 Feb 2015).

8. Swanton K. Healthy weight, healthy lives: a toolkit for developing local strategies. Department of Health, 2008. http://image.guardian. co.uk/sys-files/Society/documents/2008/10/07/heart.pdf (accessed 13 Feb 2015).

9. Hutt P, Gilmour S. Tackling inequalities in general practice: an inquiry into the quality of general practice in England. London: The King's Fund, 2010. http://www.kingsfund.org.uk/sites/files/kf/field/ field document/health-inequalities-general-practice-gp-inquiryresearch-paper-mar11.pdf (accessed 13 Feb 2015).

10. Locally Commissioned Services. PSNC Main Site. 2014. http://psnc. org.uk/services-commissioning/locally-commissioned-services/ (accessed 13 Feb 2015).

11. Todd A, Copeland A, Husband A, et al. The positive pharmacy care law: an area-level analysis of the relationship between community pharmacy distribution, urbanity and social deprivation in England. BMJ Open 2014;4:e005764.
12. Heyrman J, Spreeuwenbergh C, eds. Vocational training in general practice. Leuven: Katholieke Universiteit Leuven, 1987.

13. General Pharmaceutical Council. http://www.pharmacyregulation.org/ (accessed 13 Feb 2015)

14. Office for National Statisitics. Rural and Urban Area Definition for Lower Layer Super Output Areas. http://www.ons.gov.uk/ons/ guide-method/geography/products/area-classifications/rural-urbandefinition-and-la/rural-urban-definition-england-and-wales-/index. html (accessed 13 Feb 2015).

15. Haynes RM, Bentham CG. The effects of accessibility on general practitioner consultations, out-patient attendances and in-patient admissions in Norfolk, England. Soc Sci Med 1982;16:561-9.

16. Ritchie J, Jacoby A, Bone M. Access to primary health care. An enquiry carried out on behalf of the United Kingdom health department. London: HM Stationary Office, 1981.

17. Hopkins EJ, Pye AM, Solomon M, et al. The relation of patients age, sex and distance from surgery to the demand on the family doctor. J R Coll Gen Pract 1968;16:368-78.

18. Statistics: English indices of deprivation 2010. 2011. https://www gov.uk/government/publications/english-indices-of-deprivation-2010 (accessed 13 Feb 2015).

19. The Fuse Geo-Health Care Access Database. https://www.dur.ac uk/wolfson.institute/geohealth/ (accessed 13 Feb 2015).

20. ONS Postcode Directory Office of National Statistics. https:// geoportal.statistics.gov.uk/geoportal/catalog/main/home.page jsessionid=DA42EA7C669D7CC91FBA9C13AD511DFB (accessed 13 Feb 2015)

21. The Urban Task Force. Towards an urban renaissance. London: Routledge, 1999.

22. Lucas K, Tyler S, Christodoulou G. Assessing the 'value' of new transport initiatives in deprived neighbourhoods in the UK. Transport Policy 2009;16:115-22.

23. Hine J, Mitchell F. Better for everyone? Travel experiences and transport exclusion. Urban Stud 2001;38:319-32.

24. Duffin C. Two-week wait for GP appointments to become the norm in many practices 'within a year'. Pulse Today Published Online First: 20 May 2014. http://www.pulsetoday.co.uk/your-practice/ practice-topics/access/two-week-wait-for-gp-appointments-tobecome-the-norm-in-many-practices-within-a-year/20006739.article (accessed 13 Feb 2015).

25. Lovett A, Haynes R, Sünnenberg G, et al. Car travel time and accessibility by bus to general practitioner services: a study using patient registers and GIS. Soc Sci Med 2002;55:97-111.

26. Barr B, Bambra $C$, Whitehead $M$. The impact of NHS resource allocation policy on health inequalities in England 2001-11: longitudinal ecological study. BMJ 2014;348:g3231.

27. Pharmacy-led flu vaccination programme put on hold after GPs object. Pharmaceutical Journal. http://www.pharmaceutical-journal. com/news-and-analysis/news/pharmacy-led-flu-vaccinationprogramme-put-on-hold-after-gps-object/20066336.article (accessed 13 Feb 2015).

28. Anderson C, Thornley T. "It's easier in pharmacy": why some patients prefer to pay for flu jabs rather than use the National Health Service. BMC Health Serv Res 2014;14:35. 